This is the final peer-reviewed accepted manuscript of:

Nesci S, Pagliarani A (2019) Emerging Roles for the Mitochondrial ATP Synthase Supercomplexes. Trends in Biochemical Sciences 44:821-823. DOI: 10.1016/j.tibs.2019.07.002

The final published version is available online at: https://doi.org/10.1016/j.tibs.2019.07.002

(C) 2019. This manuscript version is made available under the Creative Commons AttributionNonCommercial-NoDerivs (CC BY-NC-ND) 4.0 International License (http://creativecommons.org/licenses/by-nc-nd/4.0/) 


\section{Emerging Roles for the Mitochondrial ATP Synthase Supercomplexes}

Salvatore $\operatorname{Nesci}^{1, *}$ and Alessandra Pagliarani ${ }^{1, *}$

1 Department of Veterinary Medical Sciences, University of Bologna, Via Tolara di Sopra, 50 - 40064 Ozzano Emilia, Bologna, Italy

*Correspondence: salvatore.nesci@unibo.it (S. Nesci) and alessandra.pagliarani@unibo.it (A. Pagliarani).

As pointed out by Gu et al. (Science 2019) in mammalian mitochondria, the H-shaped tetrameric structure of the ATP synthase, the cell powerhouse, consists of two V-shaped dimers linked by two IF1 in antiparallel arrangement. This supramolecular structure reveals new functional/ structural roles of the enzyme complex in mitochondria.

The ATP synthase is widely known as the mitochondrial enzyme complex that, under aerobic conditions, synthesizes ATP from ADP and Pi driven by Mitchell's proton motive force (pmf). In vitro studies also showed that when Gibbs' free energy of ATP phosphoanhydride bonds overwhelms the pmf, the enzyme hydrolyzes ATP and re-energizes the membrane by pumping $\mathrm{H}^{+}$in the intermembrane space. The bifunctional enzyme has two distinct domains: the hydrophilic $F_{1}$ sector

which synthesizes/hydrolyzes ATP and the hydrophobic Fo sector which translocates $\mathrm{H}^{+}$. $\mathrm{F}_{1}$ and $\mathrm{F}_{\mathrm{O}}$ are joined by a central stalk that conveys rotary motion and by a peripheral stalk that acts as a stator [1]. The ATP synthase monomers establish different contacts mediated by their peripheral stalks and $F_{O}$ subunits to form ATP synthase dimers. These dimers associate loosely into long rows by running along the edges of lamellar cristae, without requiring lipids or proteins other than ATP synthase dimers. This oligomerization locally bends the lipid bilayer, consistently with the ultrastructure of the cristae in the inner mitochondrial membrane (IMM) [2].

The recently elucidated mammalian ATP synthase structure shows that a tetramer (four monomers of ATP synthase), formed by two ATP synthase dimers that lie antiparallel to each other, is joined by two IF1 dimers [3] (Figure 1A). The active IF1 dimer forms at acid $\mathrm{pH}(\mathrm{pH}<7.0)$ and inhibits ATP hydrolysis by the ATP synthase when the pmf collapses, as it happens under some physiopathological conditions. Each IF1 in its dimeric form is associated with two $F_{1}$ moieties of two associated dimers by binding to the catalytic interface between the $\alpha_{D P}$ and $\beta_{D P}$ subunits found in loose binding conformation. Therefore, the two monomers of each dimer do not share the same IF1. This spatial arrangement allows the double function of IF1: it blocks ATP hydrolysis by a ratchet-like action on the rotor and also contributes to stabilize the inhibited state of the tetrameric enzyme structure.

The one-dimensional free-energy profile calculated by the change in free energy as a function of the interdimer distance shows that the ATP synthase dimers are kept together by the membrane itself. The dimers form a ridge in the membrane that protrudes in the matrix with negligible physical interactions [4]. Conversely, in the tetramer, two ATP synthases from adjacent dimers are bound by 
subunit-subunit interactions beyond interactions with IF1 dimers [3]. The membrane subunits arrangement of the mammalian ATP synthase is crucial to allow the interactions within ATP synthase dimers and tetramers. In general, the four monomers (I, II, III, and IV) of each tetramer participate with their own subunits in the sites of tetramer formation (Figure 1B). Two adjacent ATP synthases belonging to two different dimers form interaction sites above the IMM by their $\mathrm{k}$ and $\mathrm{b}$ subunits. In the core of the tetramer, the two dimers are linked by the opposite monomers of different dimers (i.e., II and III). The site is above the IMM between the g subunits of II and III monomers. The same monomers establish a contact site within the IMM by their e subunits.

Moreover, the structure of the interface between the ATP synthase monomers in its dimeric form reveals that the subunits that hold together the dimer in mammals differ from the ones in yeast. Accordingly, in mammals, the dimerization interface between the two Vshaped ATP synthase monomers involves the $k$ and DAPIT subunits of a monomer joined to the $g$ and $f$ subunits of the other monomer and vice versa [3] (Figure 1B). Differently, the yeast dimer is centrally maintained by a and $\mathrm{i} / \mathrm{j}$ subunit dimerization motifs and by interactions between $\mathrm{k}$ and e subunits on both sides [5]. The e and $g$ subunits, and $\mathrm{N}$-terminal helix of b subunits of each monomer in the dimer set up a 'BAR-like domain' in mammals and a 'bridgeshaped domain' in yeasts, which bends the IMM [3,5]. Noteworthy, the yeast $\mathrm{i} / \mathrm{j}$ subunit is the ortholog of mammalian DAPIT, while the yeast $\mathrm{k}$ subunit is similar to the mammalian $k$ subunit.

The mammalian 6.8-kDa proteolipid (6.8PL), absent in yeast, forms a helix that fills the central hole inside the c-ring [3]. As stated earlier, the ATP synthase dimers superassemble in long rows, forming oligomers, which are the major determinants of the edge of the cristae [6]. The ATP synthase ultrastructural organization, in which monomer pairs form dimers, dimer pairs form tetramers which in turn oligomerize in long rows, shows that subunit-subunit interactions join ATP synthase complexes and force the membrane to maintain its convexity at the apex of cristae.

The different conformational structures in the catalytic $F_{1}$ subunits (i.e., E, DP, and TP conformations) also affect the transmembrane domain, $\mathrm{F}_{\mathrm{O}}$, by imposing different rotational conformations. Flexible regions of the peripheral stalk were hypothesized to insert in both $F_{1}$ and $F_{0}$ sectors, which showed coordinated conformational changes during rotation. The central stalk undergoes torsion during catalysis, but also the c-ring shows conformational fluctuations being in contact with the a subunit which transfers protons. Thus, energy transduction coupled to proton translocation is a coordinated work of conformational adaptations of both $F_{1}$ and $F_{0}$ domains [7]. The rotor flexibility and bending involve synchronized conformational changes which generate the torsion. Moreover, the unexpected occurrence of 6.8PL within the c-ring would block the rotation of the ATP synthase [3], thus constituting another mechanical inhibitory mechanism. 
Another ATP synthase role should be considered. The enzyme's structural involvement in the permeability transition pore (PTP), which leads to cell death, is still a matter of controversy. In the clonal cell line HAP1- $\Delta(c+\delta)$, lacking both $c$ and $\delta$ subunits, the mitochondrial and nuclear-encoded enzyme subunits cannot properly associate, and the intact ATP synthase is missing with a presumptive great impact on the IMM ultrastructure. On these bases, the proven PTP formation in mitochondrial membranes devoid of ATP synthase may only apparently contrast with the putative structural involvement of the enzyme complex in the PTP. Indeed, the failed ATP synthase assembly [8] or the absence of e and g subunits [9] deeply alters the mitochondrial morphology and the formation of the cristae. It seems reasonable to think that, in the absence of the ATP synthase, as well as in the intact enzyme when $\mathrm{Ca}^{2+}$ promotes conformational changes in e and $g$ subunits, which are crucial for both the ATP synthase oligomerization and membrane bending, the IMM at the tips of cristae cannot bend and the PTP opens [10].

\section{Acknowledgments}

We thank J. Gu (Ministry of Education Key Laboratory of Protein Science, Tsinghua-Peking Joint Center for Life Sciences, Beijing Advanced Innovation Center for Structural Biology, School of Life Sciences, Tsinghua University) for making Figure 1A available. We apologize to all authors whose work could not be cited owing to space limitations.

\section{References}

Kühlbrandt, W. (2019) Structure and mechanisms of F-type ATP synthases. Annu. Rev. Biochem. 88, 515-549

2. Blum, T.B. et al. (2019) Dimers of mitochondrial ATP synthase induce membrane curvature and self-assemble into rows. Proc. Natl. Acad. Sci. U. S. A. Published online February 13, 2019. https://doi.org/10.1073/pnas.1816556116

3. Gu, J. et al. (2019) Cryo-EM structure of the mammalian ATP synthase tetramer bound with inhibitory protein IF1. Science 364, 1068-1075

4. Anselmi, C. et al. (2018) Mitochondrial ATP synthase dimers spontaneously associate due to a long-range membraneinduced force. J. Gen. Physiol. 150, 763-770

5. Guo, H. et al. (2017) Atomic model for the dimeric Fo region of mitochondrial ATP synthase. Science 358, 936-940

6. Paumard, P. et al. (2002) The ATP synthase is involved in generating mitochondrial cristae morphology. EMBO J. 21, 221230

7. Zhou, A. et al. (2015) Structure and conformational states of the bovine mitochondrial ATP synthase by cryo-EM. Elife 4, e10180

8. Carroll, J. et al. (2019) Persistence of the permeability transition pore in human mitochondria devoid of an assembled ATP synthase. Proc. Natl. Acad. Sci. U. S. A. 116, 12816-12821

9. Carraro, M. et al. (2018) High-conductance channel formation in yeast mitochondria is mediated by F-ATP synthase e and g subunits. Cell. Physiol. Biochem. 50, 1840-1855

10. Nesci, S. (2018) A lethal channel between the ATP synthase monomers. Trends Biochem. Sci. 43, 311-313 


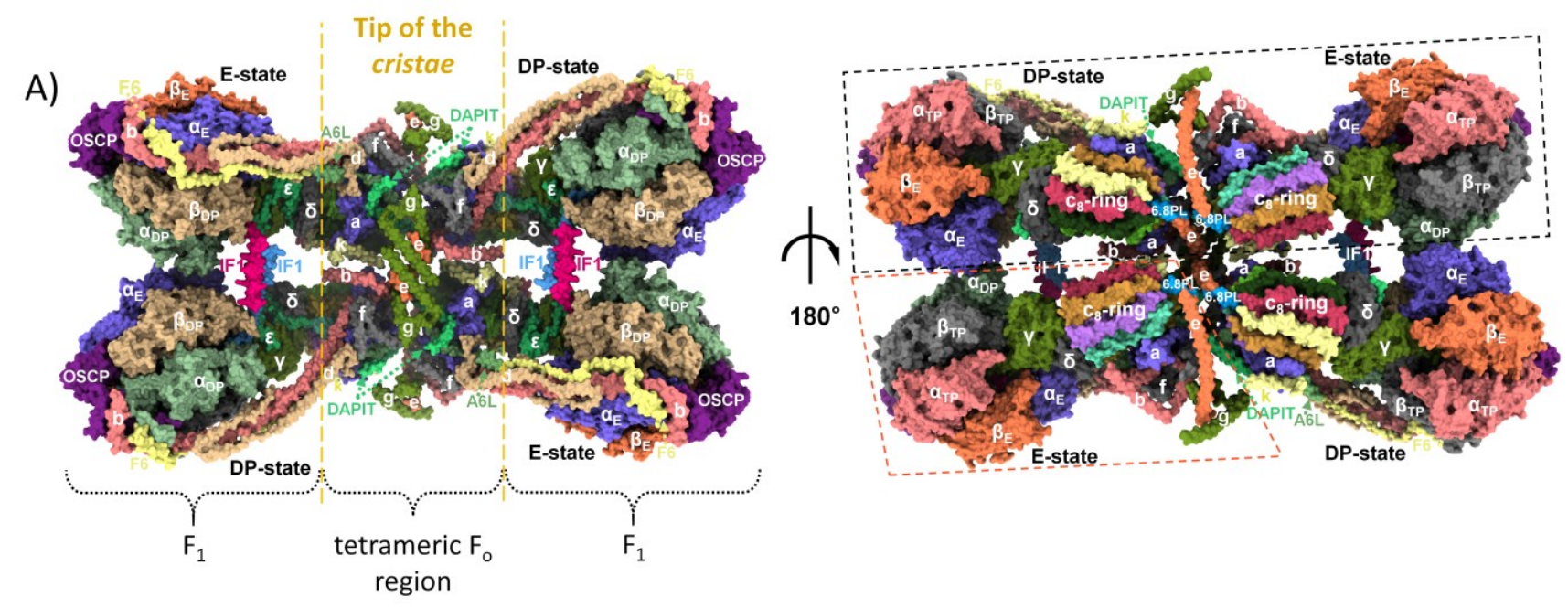

B)

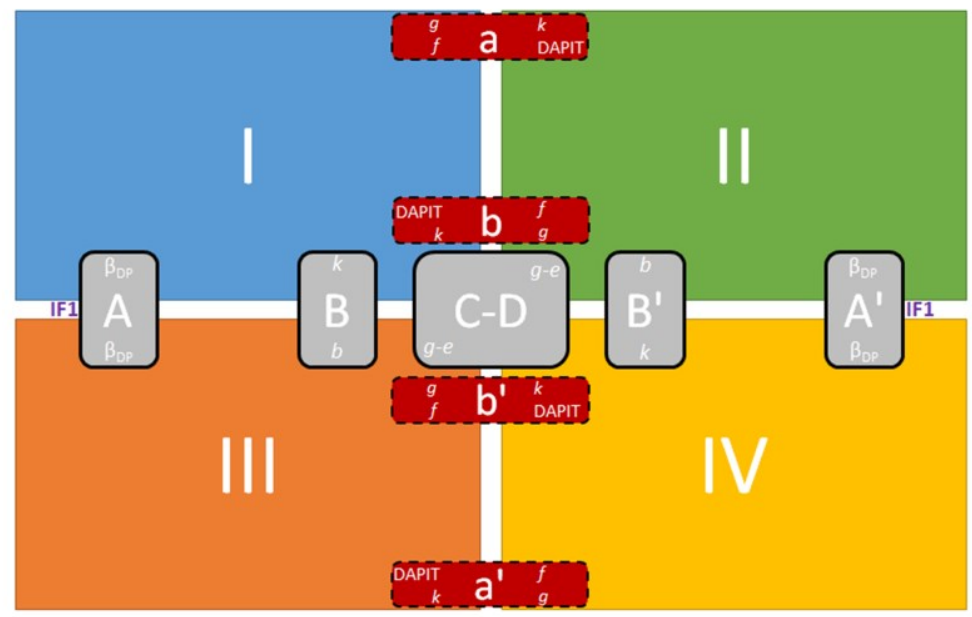

Sites involved in dimer formation

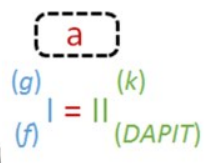

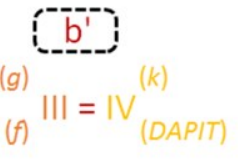

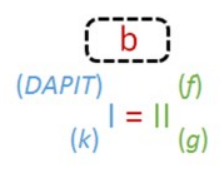

Sites above the inner membrane
Sites involved in tetramer formation

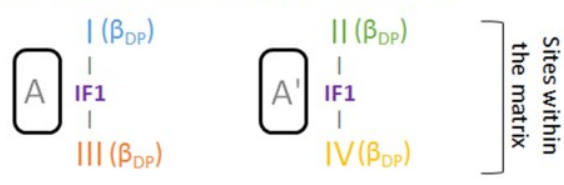

$\bigcap_{||(b)}^{\mid(k)}$
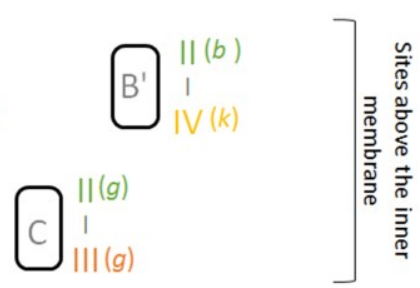

$\bigcap_{\|}^{\|(e)}$

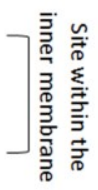

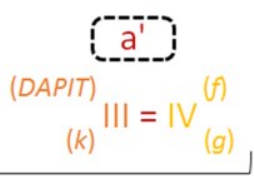

\section{西}

Figure 1. Subunit-Subunit Interactions in the Mammalian ATP Synthase Tetramer. (A) Tetrameric structure of four joined ATP synthase monomers (modified PDB ID code: 6J5K) viewed from the matrix (on the left; intramembrane Fo subunits are between yellow dashed lines representing the edge of cristae) and from intracristae space (on the right). The dashed rectangle defines one of the two dimers in the tetrameric structure, while the dashed red trapezoid highlights a monomer. (B) Left panel: the rectangles I, II, III, and IV represent the four monomers which form the tetramer, which in turn consists of two dimers (I plus II and III plus IV). The gray boxes ( $, B, C-D, B^{\prime}, A^{\prime}$ ) show the enzyme sites which join the two dimers and form the tetramer; the amaranth boxes $\left(a, b, b^{\prime}, a^{\prime}\right)$ show the intradimer interactions. The subunits involved are in white in the boxes. The right panel and the panel below show the sites in tetramer formation and in dimer formation, respectively. The identified subunits involved in the interactions are in brackets. The colors are the same as those of the monomer to which the subunits belong. For details see the text. Abbreviation: OSCP, oligomycin sensitivity conferring protein. 